Historic, archived document

Do not assume content reflects current scientific knowledge, policies, or practices. 



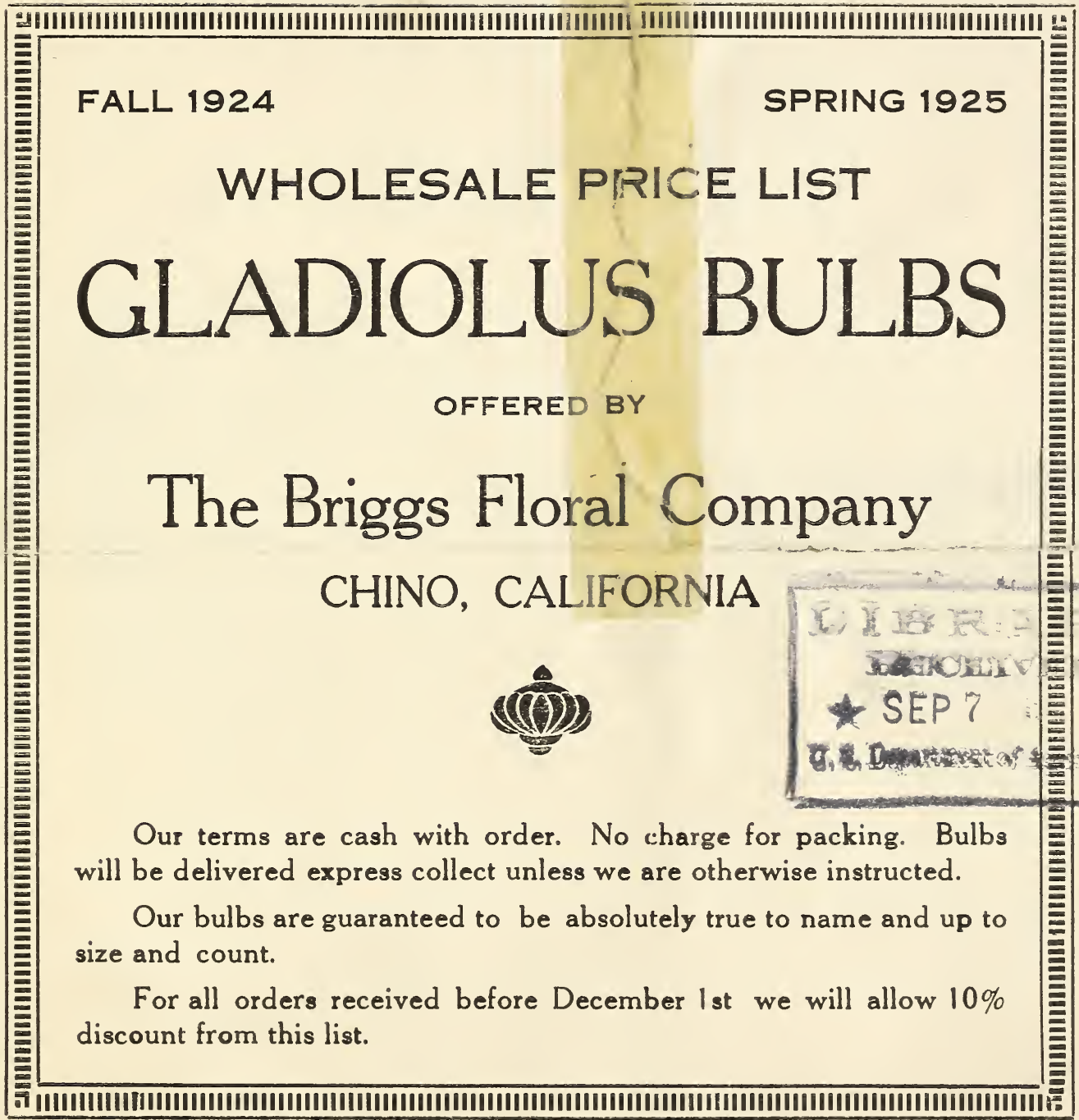




\section{Gladiolus Bulbs}

\begin{tabular}{|c|c|c|c|c|c|c|c|}
\hline & No. 1 & No. 2 & $\begin{array}{l}\text { Price } \mathrm{Pe} \\
\text { No. } 3\end{array}$ & $\begin{array}{l}\mathrm{er} 1000 \\
\text { No. } 4 \\
\end{array}$ & No. 5 & $\begin{aligned} & \mathrm{P} \\
\text { No. } 6 \quad \mathrm{~B} & \end{aligned}$ & $\begin{array}{l}\text { er } 1000 \\
\text { Bulblets }\end{array}$ \\
\hline Alice Tiplady & $\$$ & $\$$ & $\$ 25.00$ & $\$ 20.00$ & $\$ 15.00$ & $\$ 10.00$ & $\$ 3.00$ \\
\hline B. L. Smith & 50.00 & 42.00 & 34.00 & 26.00 & 18.00 & 12.00 & 2.50 \\
\hline Crimson Glow & - & 42.00 & 34.00 & 26.00 & 18.00 & 12.00 & 2.00 \\
\hline Evelyn Kirtland & 30.00 & 25.00 & 20.00 & 15.00 & 11.00 & 6.00 & 1.00 \\
\hline E. J. Shaylor & 50.00 & 42.00 & 34.00 & 26.00 & 18.00 & 12.00 & 1.00 \\
\hline Gretchen Zang & 25.00 & 21.00 & 17.00 & 14.00 & 10.00 & 6.00 & .75 \\
\hline Golden Measure & 75.00 & 63.00 & 51.00 & 39.00 & 29.00 & 15.00 & 5.00 \\
\hline Herada & 30.00 & 25.00 & 20.00 & 15.00 & 11.00 & 6.00 & .75 \\
\hline Le Marechal Foch & 25.00 & 21.00 & 17.00 & 14.00 & 10.00 & 6.00 & .75 \\
\hline Myrtle & & & 40.00 & 30.00 & 25.00 & 20.00 & 2.50 \\
\hline Mrs. Frank Pendleton & 25.00 & 21.00 & 17.00 & 14.00 & 10.00 & 6.00 & .50 \\
\hline Mary Pickford & 35.00 & 30.00 & 25.00 & 20.00 & 15.00 & 10.00 & 1.00 \\
\hline Prince of Wales & & & 20.00 & 15.00 & 11.00 & 6.00 & 1.00 \\
\hline Primunella & 80.00 & 67.00 & 54.00 & 40.00 & 28.00 & 18.00 & 5.00 \\
\hline Pythia & 75.00 & 63.00 & 51.00 & 39.00 & 29.00 & 15.00 & 5.00 \\
\hline Rose Glory & 75.00 & 63.00 & 51.00 & 39.00 & 29.00 & 15.00 & 3.00 \\
\hline Wilbrink & 25.00 & 21.00 & 17.00 & 14.00 & 10.00 & 6.00 & .50 \\
\hline
\end{tabular}


No. 1 No. 2 No. 3 No. 4 No. 5 No. 6 Bulblets

American Beauty

Anna Eberius

A. B. Kunderd

Angola

Argo

Altair

Baron Hulot

Beaconflame

Carmen Sylva

Dorothy Wheeler

Elizabeth Tabor

Gold Drop

Helen Franklin

Jewell

Kunderds Foch

Mrs. Dr. Norton

Mary Fennel

Marie Kunderd

Mrs. F. C. Peters

Peach Rose

Pride of Lancaster

Purple Glory

R. J. Kunderd

Scarlano

Sweet Lavendar
$\$ 18.00 \quad \$ 15.00 \quad \$ 12.00 \quad \$ 9.00 \quad \$ 7.00 \quad \$ 5.00 \quad \$ 10.00$

\begin{tabular}{lrrr}
$41.00 \quad 30.00 \quad 20.00 \quad 3.00$ \\
\hline
\end{tabular}

\begin{tabular}{lllllll}
20.00 & 17.00 & 14.00 & 11.00 & 9.00 & 7.00 & 10.00 \\
\hline
\end{tabular}

\begin{tabular}{llll}
1.50 & 1.10 & .60 & 1.00 \\
\hline
\end{tabular}

\begin{tabular}{llll}
2.60 & 1.80 & 1.20 & 1.00 \\
\hline
\end{tabular}

\begin{tabular}{llll}
2.10 & 1.60 & 1.10 & 1.00 \\
\hline
\end{tabular}

\begin{tabular}{lllll}
4.00 & 3.00 & 2.50 & 2.00 & 3.00 \\
\hline
\end{tabular}

\begin{tabular}{lllllll}
5.00 & 4.20 & 3.40 & 2.60 & 1.80 & 1.20 & 1.00 \\
\hline
\end{tabular}

\begin{tabular}{lllllll}
20.00 & 17.00 & 14.00 & 10.00 & 8.00 & 6.00 & 15.00 \\
\hline
\end{tabular}

\begin{tabular}{lllllll}
5.00 & 4.20 & 3.40 & 2.60 & 1.80 & 1.20 & 2.00 \\
\hline
\end{tabular}

\begin{tabular}{lllllll}
20.00 & 17.00 & 14.00 & 10.00 & 8.00 & 6.00 & 15.00 \\
\hline
\end{tabular}

\begin{tabular}{l}
$3.00 \quad 2.50 \quad 2.00 \quad 2.00$ \\
\hline
\end{tabular}

\begin{tabular}{llllll}
3.30 & 2.60 & 2.10 & 1.60 & 1.10 & 2.00 \\
\hline
\end{tabular}

$\begin{array}{lllllll}20.00 & 17.00 & 14.00 & 10.00 & 8.00 & 6.00 & 15.00\end{array}$

\begin{tabular}{lllllll}
20.00 & 17.00 & 14.00 & 10.00 & 8.00 & 6.00 & 15.00 \\
\hline
\end{tabular}

\begin{tabular}{llll}
2.00 & $1.50 \quad 1.00 \quad 1.00$ \\
\hline
\end{tabular}

$\begin{array}{llll}3.00 & 2.50 & 2.00 & 2.00\end{array}$

\begin{tabular}{lllllll}
50.00 & 42.00 & 34.00 & 26.00 & 18.00 & 12.00 & 50.00 \\
\hline
\end{tabular} \begin{tabular}{lllllll}
40.00 & 34.00 & 27.00 & 22.00 & 17.00 & 12.00 & 25.00 \\
\hline
\end{tabular} \begin{tabular}{lllllll}
35.00 & 30.00 & 25.00 & 20.00 & 15.00 & 10.00 & 20.00 \\
\hline
\end{tabular}

\begin{tabular}{llll}
2.60 & 1.80 & $1.20 \quad 2.00$ \\
\hline
\end{tabular}

\begin{tabular}{lllllll}
20.00 & 17.00 & 14.00 & 11.00 & 9.00 & 7.00 & 10.00 \\
\hline
\end{tabular}
\begin{tabular}{lllllll}
20.00 & 17.00 & 14.00 & 11.00 & 9.00 & 7.00 & 10.00 \\
\hline
\end{tabular}

\begin{tabular}{lllllll}
5.00 & 4.20 & 3.40 & 2.60 & 1.80 & 1.20 & 2.00 \\
\hline
\end{tabular}

\begin{tabular}{lllllll}
25.00 & 21.00 & 17.00 & 14.00 & 10.00 & 8.00 & 20.00 \\
\hline
\end{tabular}
25 at 100 Price-Less than 25 add $10 \%$ 
